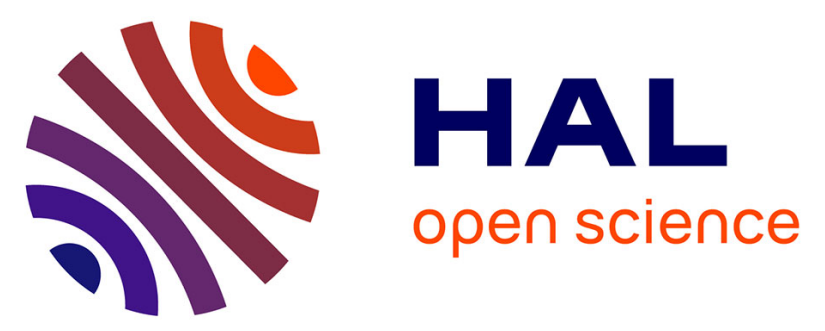

\title{
In vivo evidence for transintestinal cholesterol efflux in patients with complete common bile duct obstruction
}

François Moreau, Claire Blanchard, Christophe Perret, Laurent Flet, Frédéric

Douane, Eric Frampas, Eric Mirallie, Mikael Croyal, Audrey Aguesse, Michel

Krempf, et al.

\section{To cite this version:}

François Moreau, Claire Blanchard, Christophe Perret, Laurent Flet, Frédéric Douane, et al.. In vivo evidence for transintestinal cholesterol efflux in patients with complete common bile duct obstruction. Journal of clinical lipidology, 2019, 13 (1), pp.213-217.e1. 10.1016/j.jacl.2018.09.010 . hal-03097135

\section{HAL Id: hal-03097135 \\ https://hal.science/hal-03097135}

Submitted on 5 Jan 2021

HAL is a multi-disciplinary open access archive for the deposit and dissemination of scientific research documents, whether they are published or not. The documents may come from teaching and research institutions in France or abroad, or from public or private research centers.
L'archive ouverte pluridisciplinaire HAL, est destinée au dépôt et à la diffusion de documents scientifiques de niveau recherche, publiés ou non, émanant des établissements d'enseignement et de recherche français ou étrangers, des laboratoires publics ou privés. 


\section{In vivo evidence for transintestinal cholesterol efflux in patients with complete common bile duct obstruction}

François Moreau ${ }^{1 *} \mathrm{PhD}$; Claire Blanchard $1,2^{\star} \mathrm{MD}, \mathrm{PhD}$; Christophe Perret ${ }^{3} \mathrm{MD}$; Laurent Flet $^{4}$ PharmD ; Frédéric Douane ${ }^{3}$ MD ; Eric Frampas ${ }^{3}$ MD ; Eric Mirallie ${ }^{2}$ MD ; Mikael Croyal5, 6 PhD ; Audrey Aguesse ${ }^{5,6}$, Michel Krempf ${ }^{5,6}$ MD, PhD ; Xavier Prieur ${ }^{1} \mathrm{PhD}$; Matthieu Pichelin ${ }^{1,7}$ PharmD ; Bertrand Cariou ${ }^{1,7^{*}} \mathrm{MD}, \mathrm{PhD} \&$ Cédric Le May ${ }^{1 *} \# \mathrm{PhD}$.

* contributed equally

Running head: TICE is active in humans

\section{Affiliations}

1L'institut du thorax, INSERM, CNRS, UNIV Nantes, Nantes, France; 2 Service de Clinique de Chirurgie Digestive et Endocrinienne, CHU de Nantes, France; ${ }^{3}$ Department of Radiology, $\mathrm{CHU}$ Nantes, Nantes, France; ${ }^{4}$ Pharmacie, CHU Hôtel Dieu, 44093 Nantes Cedex, France; 5INRA, UMR 1280, Physiologie des Adaptations Nutritionnelles, CHU Nantes, France ; ${ }^{6} \mathrm{CRNHO}$, West Human Nutrition Research Center, CHU, F-44093 Nantes, France; ' 'L'institut du thorax, INSERM, CNRS, UNIV Nantes, CHU Nantes, Nantes, France

Corresponding author mail id: : cedric.lemay@univ-nantes.fr

Contact information for the corresponding author ::

Cedric Le May, PhD,

l'institut du thorax,

INSERM, CNRS, UNIV Nantes,

Nantes - France.

Abbreviations used in this paper: MRI, Magnetic Resonance Imaging; ND, Non-detectable; TICE, Trans Intestinal Cholesterol Efflux 


\section{Abstract}

Background: Beyond the hepatobiliary pathway, studies have demonstrated that direct TransIntestinal Cholesterol Efflux (TICE) of plasma-derived cholesterol may contribute to reverse cholesterol transport. The clinical evidence of TICE in human remains challenged due to the difficulty to discriminate the hepatobiliary and transintestinal routes in vivo.

Objective: To provide the first proof of concept that TICE exists in vivo in humans by demonstrating that plasma labeled cholesterol can be excreted in the feces of patients with complete bile duct obstruction.

Methods: Plasma, bile and fecal cholesterol excretion was measured by mass spectrometry 24,48 and $72 \mathrm{~h}$ after intravenous injection of D7-cholesterol in two patients presenting cholangiocarcinomas with a total obstruction of their primary bile duct.

Results: No trace of bile acids was detected in the feces of the two patients. Despite this, a significant amount of plasma D7-cholesterol was quantified in the feces of the two patients $48 \mathrm{~h}$ and $72 \mathrm{~h}$ after the intravenous injection.

Conclusion: our data bring a direct proof that TICE is an active pathway in humans.

ClinicalTrials.gov Registration number: NCT01958216

\section{Highlights:}

The transintestinal (TICE) route is crucial for plasma cholesterol excretion in rodent To date, the physiological relevance of TICE in humans remains questioned Patients with obstructed biliary route can excrete plasma cholesterol in their feces TICE is an active pathway in humans

Keywords : Cholesterol, Trans Intestinal Cholesterol Efflux, Humans, Bile duct obstruction 


\section{Introduction}

Until recently, the hepatobiliary pathway was described as the sole route involved in the fecal elimination of plasma cholesterol and reverse cholesterol transport (reviewed in 1-3). In 2007, Groen's team first demonstrated the existence in mice of a new pathway of cholesterol excretion named TICE for TransIntestinal Cholesterol Efflux ${ }^{4}$. Although molecular mechanisms remain largely unknown, several studies have contributed to a better understanding of this pathway ${ }^{4-11}$. While TICE appears to be twice more efficient than the biliary pathway to excrete plasma-derived cholesterol in mice ${ }^{6}$, the relative importance and more importantly the clinical relevance of TICE in human remains a crucial but open question. Several indirect evidences have nonetheless suggested the existence of TICE in human ${ }^{12,13}$. In 1959, Cheng and Stanley first described an intestinal cholesterol secretion in three patients presenting a total obstruction of the biliary and pancreatic ducts ${ }^{12}$. Later, Simmonds et al performed intestine perfusions in humans and suggested that $44 \%$ of the cholesterol found in the intestinal lumen derived from intestinal cells ${ }^{13}$. The main criticism for these studies regarding the TICE existence is the lack of evidence that the cholesterol excreted in the intestinal lumen comes directly from the plasma. In 2013, we demonstrated with Ussing chambers that human jejunal biopsies actively excrete cholesterol from basolateral to luminal chambers, suggesting that TICE is operative and inducible in human ${ }^{8}$.

Our proof-of-concept study aims to demonstrate by using cholesterol tracer that patients with a defective hepatobiliary pathway can excrete plasma-derived cholesterol in their feces.

\section{Materials and methods}

\section{Clinical protocol:}

The « In vivo TICE » study was a single-centre prospective interventional study and was conducted between 2014 and 2016 at the Digestive and Endocrine Surgery department of Nantes University Hospital and registered in clinicaltrials.gov as study NCT01958216. It was a single-centre prospective interventional study conducted between 2014 and 2016 at the Digestive and Endocrine Surgery department of Nantes University Hospital. The goal of this proof of concept study was to provide the first proof of the TransIntestinal Cholesterol Efflux (TICE) existence in humans by using stable isotopes in patients with bile duct diversion. The study was approved by the ethics committee (IRB/IEC) CPP Ouest IV ( $\left.{ }^{\circ} 08 / 12\right)$ and the ANSM - French National Agency for Medicines and Health Products Safety ( $n^{\circ}$ 2012-A01666-35). The Inclusion criteria were adult patients (aged $\geq 18$ years) presenting a total obstruction of the intestinal biliary flow with an external biliary derivation. We excluded from the study patients with inflammatory bowel disease, aphagia, renal or hepatocellular insufficiency, primary intestinal tumor, cholangitis or severe sepsis, acute or chronic diarrhea or under pregnancy. The biliary pathway obstruction was initially validated by magnetic 
resonance imaging before the set-up of the external biliary cannulae. After signing the clinical consent, plasma, biliary and fecal samples were harvested in patients at day 0 . Then, bile diverted patients have received an intravenous injection of deuterated cholesterol diluted in intralipid $20 \%$. The plasma, biliary and fecal content of deuterated cholesterol will be measured, by mass spectrometry, at 24, 48 and 72 hours after the initial input.

\section{Mass spectrometry measurement:}

The detailed protocols are available as supplemental file. 


\section{Results}

We recruited two patients with neoplasia of the root biliary duct (i.e. cholangiocarcinomas). Their clinical characteristics are listed in Table 1.

Table 1: Clinical characteristics of study subjects

\begin{tabular}{|c|c|c|c|}
\hline & Patient 1 & Patient 2 & $\begin{array}{l}\text { Reference } \\
\text { ranges }\end{array}$ \\
\hline Age (Years) & 84 & 66 & \\
\hline Gender & Female & Male & \\
\hline Body weight (kg) & 62.4 & 69 & \\
\hline Hemoglobin (g/dL) & 10.9 & 11.9 & $13,5-17,5$ \\
\hline Red blood cells (Tera/L) & 3.67 & 3.71 & $4,5-5,9$ \\
\hline Leukocytes (Giga/L) & 12.9 & 8.96 & $4-10$ \\
\hline Platelets (Giga/L) & 333 & 267 & $150-400$ \\
\hline $\mathrm{AST}^{*}(\mu \mathrm{kat} / \mathrm{L})$ & 1.37 & 1.23 & $0-0,85$ \\
\hline $\mathrm{ALT}^{*}(\mu \mathrm{kat} / \mathrm{L})$ & 1.43 & 1.26 & $0-0,85$ \\
\hline $\mathrm{GGT}^{*}(\mu \mathrm{kat} / \mathrm{L})$ & 0.61 & 9.17 & $0-1,19$ \\
\hline $\mathrm{APL}^{*}(\mu \mathrm{kat} / \mathrm{L})$ & 2.33 & 4.91 & $0,92-2$ \\
\hline Albumin (g/L) & 25.1 & 31 & $35-52$ \\
\hline Creatinine $(\mu \mathrm{M})$ & 70 & 62 & $62-106$ \\
\hline Bilirubin $(\mu \mathrm{M})$ & 346 & 367 & $0-21$ \\
\hline \multicolumn{4}{|l|}{ Conjugated Bilirubin } \\
\hline$(\mu \mathrm{M})$ & 283 & 327 & $0-5$ \\
\hline Glucose (mM) & 5.4 & 4.7 & $4,6-6,4$ \\
\hline \multicolumn{4}{|l|}{ Total Cholesterol } \\
\hline$(\mathrm{mg} / \mathrm{dL})$ & 395 & 451 & $82-197$ \\
\hline HDL Cholesterol (mg/dL) & 8 & 8 & $42-68$ \\
\hline Triglycerides (mg/dL) & 345 & 343 & $44-149$ \\
\hline \multicolumn{4}{|c|}{$\begin{array}{l}\text { * AST: aspartate aminotransferase ; ALT: alanine aminotransferase ; } \\
\text { GGT: Gamma-Glutamyl Transferase : APL: alkaline phosphatase level. }\end{array}$} \\
\hline
\end{tabular}

As a primary step, we verified and confirmed the complete obstruction of biliary pathways by bileduct magnetic resonance imaging (Figure $1 \mathrm{~A}$ ). Next, we confirmed by mass spectrometry analysis that lithocholic acid, the most abundant bile acid in the human feces, was undetectable in the feces of the two patients (data not shown). In addition, the use of a colorimetric enzymatic assay confirmed 
the absence of any bile acid species in the feces (data not shown). Together these results established that these two patients had a complete biliary duct obstruction, thereby ruling out the possibility for a biliary delivery of plasma-derived cholesterol in the gut.

After the set-up of the external biliary drainage, we harvested blood, bile and feces samples prior an intravenous injection of D7-cholesterol (100mg) and 10 minutes, $24 \mathrm{~h}, 48 \mathrm{~h}$ and $72 \mathrm{~h}$ after the injection. We measured a significant amount of plasma D7-cholesterol in both patients ten minutes after injection (Figure 1B) with a subsequent reduction of D7-cholesterol levels during the three following $s$ validating the efficiency of circulating cholesterol clearance pathways. We also quantified D7cholesterol in the bile collected via the external cannula (Patient 1: 0.28 mg, 0,29 mg and 0,76 mg respectively excreted during day $1,2 \& 3$; Patient $2: 0.38 \mathrm{mg}, 1.37 \mathrm{mg}$ and $0.43 \mathrm{mg}$ respectively at day 1,2 \& 3$)$.

Interestingly, despite a total obstruction of the biliary route, a significant amount of D7-cholesterol was detected in the feces of the two patients $48 \mathrm{~h}$ and $72 \mathrm{~h}$ after injection. Fecal D7-cholesterol was under the limit of detection in both patients $24 \mathrm{~h}$ after the injection. Regarding patient 1 , we observed the appearance of the D7-cholesterol peak at day-2 (Figure 1C) and quantified 0,13 $\mu \mathrm{g}$ of D7cholesterol per gram of feces (Figure 1B). Patient 1 did not produce any feces at day-3. We observed a similar pattern with patient 2. Although, he did not produce feces at day-2, we quantified $0,33 \mu \mathrm{g}$ of D7-cholesterol per gram of feces at day-3 (Figure 1B\&C).

\section{Discussion}

These data constituted the first direct proof that plasma-derived cholesterol can be excreted in the feces of patients with a complete biliary duct obstruction, thereby validating that TICE is operative in vivo in humans.

By contrast with two previous studies ${ }^{12,13}$, the use of a cholesterol tracer allow us to specifically follow the fecal elimination of cholesterol from the plasma compartment. Interestingly, while we were completing this study, Groen's team published an in vivo cholesterol kinetic study performed in humans and showed by modeling cholesterol fluxes that TICE could contribute to $30-35 \%$ of the cholesterol excreted in the feces under basal condition and can be strongly upregulated with ezetimibe $^{9}$. Despite its novelty, some elements of our study can be opened for discussion. The design of the study focuses on few patients to provide a proof of concept evidence that plasma cholesterol can be eliminated in the feces of individuals with obstructed biliary pathway. Clearly, the very small sample size is a limitation and additional experiments with more subjects are needed to precise the biliary and transintestinal flow measurement. Two independent pathways can contribute to the intestinal excretion of plasma cholesterol. TICE is an active pathway requiring several transporters both at the basolateral and apical sides of enterocytes (reviewed in ${ }^{3,14}$ ). The rapid turnover of the epithelial intestinal cells can also contribute to a part of the clearance of the plasma- 
derived D7-cholesterol after its active uptake from the plasma. This phenomenon named shedding is difficult to quantify without intrusive methods that cannot be used in humans. In mouse models under unstimulated or stimulated conditions, shedding cells contribute respectively from 10 to $30 \%$ of the cholesterol excreted in the feces ${ }^{4,15}$. We cannot discard the possibility that a part of the fecal D7-cholesterol originates from desquamated cells, a phenomenon that we can call "passive" TICE. For practical and logistical reasons but also because of the precarious health of our frail patients, we did not succeed to harvest their full feces production during the three days after injection. This would have been very helpful for estimating the relative importance of the hepatobiliary versus the transintestinal routes in plasma cholesterol elimination, as previously shown in mice and humans with mathematical models ${ }^{6,9}$. However, if we assume that adult humans excrete about $200 \mathrm{~g}$ of feces per day, we can extrapolate that at day-2, patient 1 excretes $297,5 \mu \mathrm{g}$ of biliary D7-cholesterol and $26 \mu \mathrm{g}$ of D7-cholesterol via the transintestinal route; at day-3, patient 2 excretes $432 \mu \mathrm{g}$ of biliary D7cholesterol and $66 \mu \mathrm{g}$ of D7-cholesterol via TICE. Based on these calculations, TICE appears to be six- to ten-fold less efficient than the hepatobiliary pathway to excrete plasma cholesterol. This low transintestinal efflux of plasma cholesterol can be explained, at least partly, by the complete obstruction/derivation of their biliary ducts. Indeed, hepatobiliary and transintestinal routes are two pathways that exist independently but TICE is strongly stimulated by cholesterol acceptors (phospholipids and bile acids) provided by the bile flow ${ }^{4,5}$. Van der Velde et al reported that intestinal cholesterol excretion could occur in the absence of bile salts if luminal phospholipids are available ${ }^{4}$. We propose that the intestinal flow of D7-cholesterol excreted may be far from maximal in our patients due to the lack of bile acids as acceptors in the lumen and probably made possible by the luminal phospholipids provided by the diet.

To date, TICE remains a poorly characterized pathway and only few intestinal molecular players have been yet identified in mice as important for TICE regulation, among which apical transporters such as $A B C G 5 / G 8^{6}, A B C B 1^{8}$ and $\mathrm{NPC} 1{ }^{2} 1^{9}$ and also the $\mathrm{LDL}$ receptor located the basolateral side of enterocytes ${ }^{8}$ (Figure 2). Interestingly, these molecular determinants have already been associated with cardiovascular diseases in several Mendelian randomization studies ${ }^{16-18}$. However, these transporters are not only located in intestinal cells but also in the liver and thus the direct evidence that a defective TICE can promote cardiovascular disease progression remains to be established. An initial step was made when Ryan Temel and colleagues provide the first evidence that TICE was an active component of the Reverse Cholesterol Transport (RCT) pathway in mice ${ }^{7}$. The RCT route originally described by John Glomset in 1968, allows the efflux of cholesterol from peripheral cells to the HDL particles and its transport to the liver for fecal elimination (reviewed in ${ }^{1-}$ $\left.{ }^{3}\right)$. Due to its ability to reduce cholesterol content in macrophages located in the arterial walls, RCT has been considered for long time as an anti-atherogenic pathway (reviewed in ${ }^{1-3}$ ). If TICE is an active and final component of the RCT, its stimulation should delay atherosclerosis progression. Generating specific pharmaceutical TICE modulators will be required to validate this hypothesis. 
Statins and ezetimibe are widely used in clinical practice to decrease plasma cholesterol respectively by affecting cholesterol synthesis and intestinal cholesterol absorption. Interestingly, these drugs also stimulate TICE in vivo either in mice ${ }^{8,9}$ or in humans ${ }^{9}$. In addition, some preclinical data in mice suggest that FXR agonist (PX20606) can stimulate TICE, at least by increasing the hydrophilicity of the bile salt pool ${ }^{11}$. However, to the best of our knowledge, no drugs that specifically target TICE are currently in development but our data bring an additional but direct proof that TICE is an active pathway in humans and suggest that a better understanding of the molecular mechanisms behind this efflux could open new therapeutic options for patients at risk of cardiovascular diseases.

\section{Conflicts of interest}

These authors disclose no conflicts.

\section{Acknowledgments}

This work was supported by a clinical research internal grant (RC11_0008) from the University Hospital of Nantes and by "la region des Pays de la Loire".

\section{Author contributions}

François Moreau and Claire Blanchard collected and analyzed the data and revised the manuscript, Laurent Flet prepared the D7-cholesterol in intralipid solution; Christophe Perret, Frédéric Douane, Eric Frampas performed the Magnetic Resonance Imaging of the primary bile ducts; Mikael Croyal, Audrey Aguesse and Michel Krempf contributed to the Mass spectrometry analyses; Xavier Prieur and Eric Miraille revised the manuscript; Matthieu Pichelin designed the research study and supervised the clinical operations and the data collection; Bertrand Cariou \& Cedric Le May designed the research study supervised the study, analysed the data, wrote and edited the manuscript. All of the authors approved the final version of the manuscript.

\section{References:}

1. Rader DJ, Alexander ET, Weibel GL, et al. The role of reverse cholesterol transport in animals and humans and relationship to atherosclerosis. J. Lipid Res. 2009. S189-S194. 
2. Hellerstein M, and Turner S. Reverse cholesterol transport fluxes. Curr Opin Lipidol 2014 ; 25:4047

3. Temel RE, and Brown JM. A new model of reverse cholesterol transport: enTICEing strategies to stimulate intestinal cholesterol excretion. Trends Pharmacol Sci 2015; 36: 440-451.

4. van der Velde $\mathrm{AE}$, Vrins $\mathrm{C}$, van den Oever $\mathrm{K}$, et al. Direct Intestinal Cholesterol Secretion Contributes Significantly to Total Fecal Neutral Sterol Excretion in Mice. Gastroenterology 2007; 133: 967-975.

5. Van der Velde AE, Vrins $C$, Van den Oever K, et al. Regulation of direct transintestinal cholesterol excretion in mice. Am J Physiol Gastrointest Liver Physiol 2008; 295: G203-208.

6. van der Veen JN, van Dijk TH, Vrins C, et al. Activation of the Liver X Receptor Stimulates Transintestinal Excretion of Plasma Cholesterol. J Biol Chem 2009; 284: 19211-19219.

7. Temel RE, Sawyer JK, Yu L, et al. Biliary Sterol Secretion Is Not Required for Macrophage Reverse Cholesterol Transport. Cell Metab 2010; 12: 96-102.

8. Le May C, Berger J, Lespine A, et al. Transintestinal Cholesterol Excretion Is an Active Metabolic Process Modulated by PCSK9 and Statin Involving ABCB1. Arterioscler Thromb Vasc Biol2013; 33: 1484-1493.

9. Jakulj L, van Dijk TH, de Boer JF, et al. Transintestinal Cholesterol Transport Is Active in Mice and Humans and Controls Ezetimibe-Induced Fecal Neutral Sterol Excretion. Cell Metab 2016; 24 : $1-12$.

10. Blanchard C, Moreau F, Ayer A, et al. Roux-en-Y gastric bypass reduces plasma cholesterol in diet-induced obese mice by affecting trans-intestinal cholesterol excretion and intestinal cholesterol absorption. Int J Obes (Lond) 2017.

11. de Boer JF, Schonewille M, Boesjes M, et al. Intestinal Farnesoid X Receptor Controls Transintestinal Cholesterol Excretion in Mice. Gastroenterology 2017; 152: 1126-1138.e6.

12. Cheng SH and Stanley M. Secretion of cholesterol by intestinal mucosa in patients with complete bile duct obstruction. Proc Soc Exp Biol Med 1959; 101: 223-225.

13. Simmonds WJ, Hofmann AF and Theodor E. Absorption of Cholesterol from a Micellar Solution: Intestinal Perfusion Studies in Man. J Clin Invest 1967; 46: 874-890.

14. Reeskamp LF, Meessen ECE and Groen AK. Transintestinal cholesterol excretion in humans. Curr Opin Lipidol 2018; 29(1):10-17.

15. Férézou J, Coste $T$ and Chevallier F. Origins of neutral sterols in human feces studied by stable isotope labeling ( $\mathrm{D}$ and $13 \mathrm{C}$ ). Existence of an external secretion of cholesterol. Digestion 1981; 21 : 232-243.

16. Lotta LA, Sharp SJ, Burgess S, et al. Association Between Low-Density Lipoprotein CholesterolLowering Genetic Variants and Risk of Type 2 Diabetes : A Meta-analysis. JAMA 2016; 316(13): 1383-1391. 
17. Ross S, D'Mello M, Anand SS, et al. Effect of Bile Acid Sequestrants on the Risk of Cardiovascular Events, A Mendelian Randomization Analysis. Circ Cardiovasc Genet 2015; 8 : 618627.

18. Ference BA, Ginsberg HN, Graham I, et al. Low-density lipoproteins cause atherosclerotic cardiovascular disease. 1. Evidence from genetic, epidemiologic, and clinical studies. A consensus statement from the European Atherosclerosis Society Consensus Panel. Eur Heart J. 2017 Aug $21 ; 38(32): 2459-2472$. 
A

Patient 1

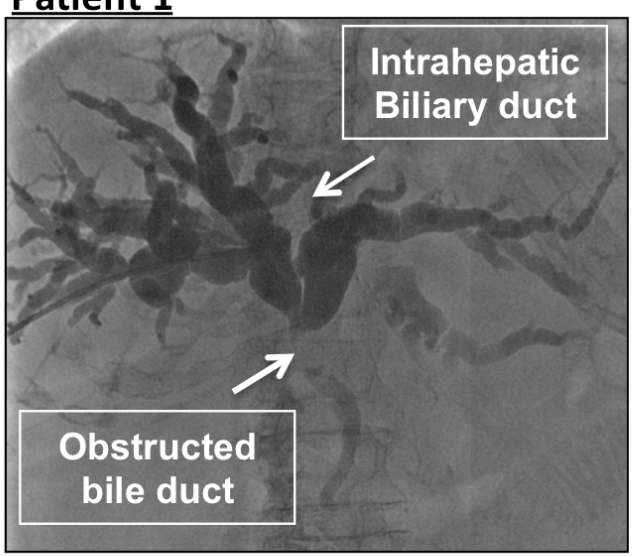

Patient 2

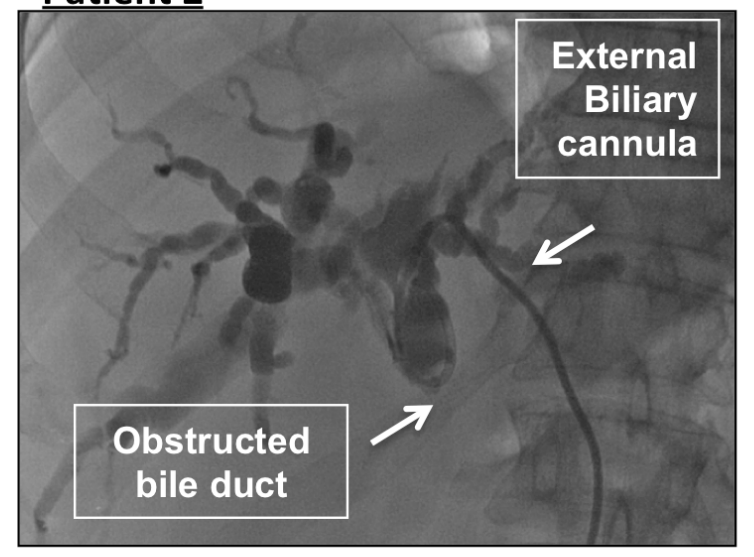

B

\begin{tabular}{|c|c|c|c|c|c|c|c|}
\hline & & Prior Injection & +10 minutes & $+24 h$ & $+48 h$ & $+72 h$ & \\
\hline \multirow[t]{2}{*}{ Plasma } & Patient 1 & 0 & 7,67 & 6,12 & 6,43 & 4,88 & $\mathrm{ug} / \mathrm{mL}$ \\
\hline & Patient 2 & 0 & 15,04 & 12,35 & 11,63 & 8,81 & \\
\hline \multirow[t]{2}{*}{ Bile } & Patient 1 & 0 & - & 1,28 & 2,48 & 2,54 & $\mathrm{ug} / \mathrm{mL}$ \\
\hline & Patient 2 & 0 & - & 0,38 & 0,76 & 0,27 & \\
\hline \multirow[t]{5}{*}{ Feces } & Patient 1 & 0 & - & ND & 0,13 & No feces & ug/g feces \\
\hline & Patient 2 & 0 & - & ND & No feces & 0,33 & \\
\hline & & & Bile volume $(\mathrm{mL})$ & $0-24 h$ & $24-48 h$ & $48-72 h$ & \\
\hline & & & Patient 1 & 220 & 120 & 300 & \\
\hline & & & Patient 2 & 1000 & 1800 & 1600 & \\
\hline
\end{tabular}

C


\section{Legends}


Figure 1: A) Magnetic Resonance Imaging of the primary bile duct of study subjects; B) Plasma, bile and fecal concentration of D7-cholesterol measured at indicated time points; C) Mass spectrometry spectra showing the fecal appearance of D7-cholesterol of patients $1 \& 2$.

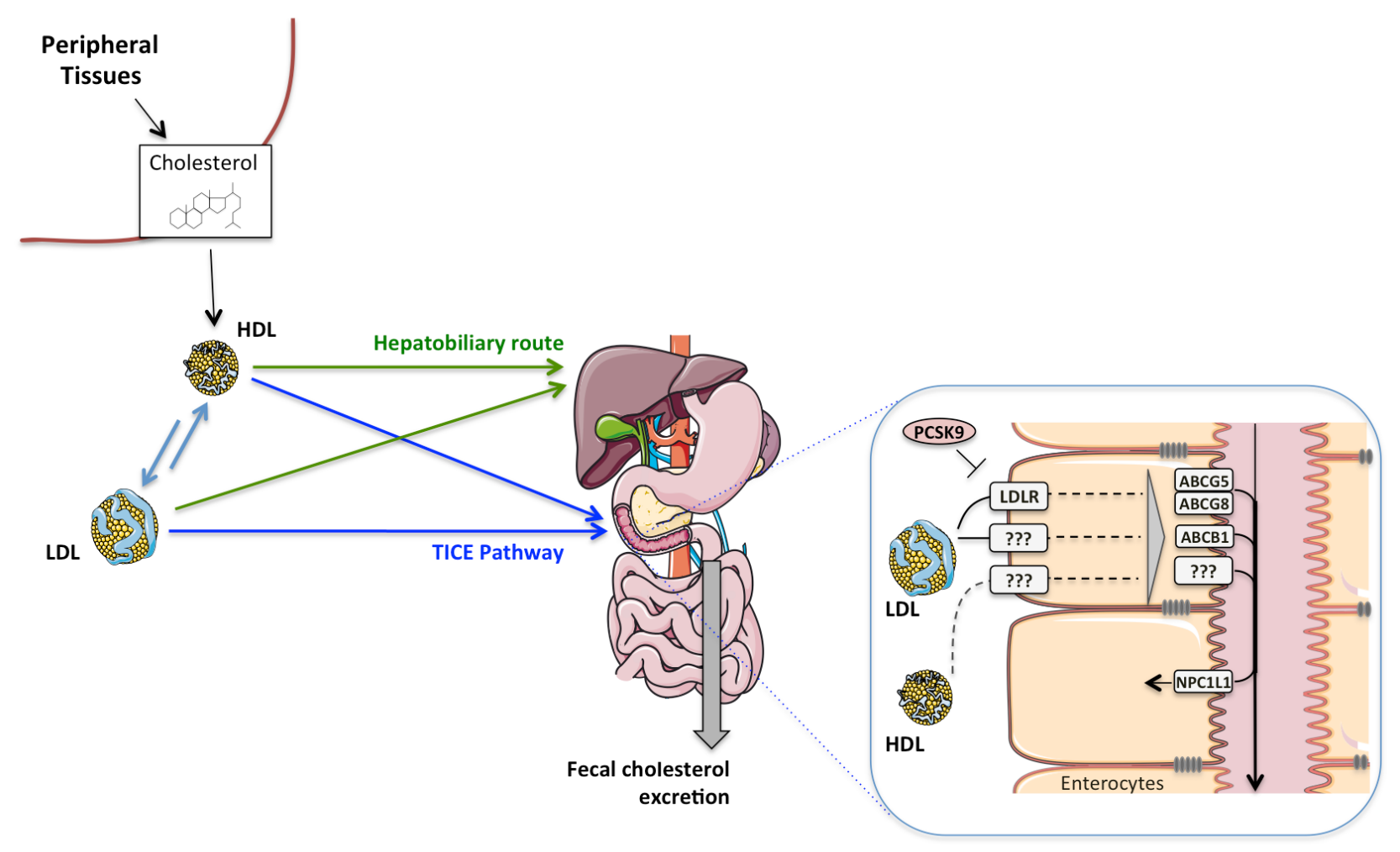

Figure 2: TICE and Reverse Cholesterol Transport. Cholesterol is effluxed from peripheral cells to HDL (high-density lipoprotein). After its transfer or not to LDL (low-density lipoprotein), cholesterol can be eliminated in the intestinal lumen for fecal elimination via the hepatobiliary or the transintestinal pathway. At the basolateral side of intestinal cells, cholesterol from LDL and HDL can be internalized by the LDL receptor (LDLR) and other unidentified transporters. Intracellularly, mechanisms involved in the cholesterol traffic across the enterocytes remains to date unexplored. At the brush border, $A B C G 5 / G 8$ and $A B C B 1$ are both responsible for the luminal excretion of cholesterol when NPC1L1 limits TICE by reabsorbing luminal cholesterol. 